5 Goldberg JA, Willmott N, Kerr DJ, Sutherland C, McArdle CA. An in vivo assessment of adriamycin-loaded albumin microspheres. Br F Cancer 1992; 65: 393-5.

6 Booser DJ, Hortobagyi GN. Treatment of locally advanced breast cancer. Semin Oncol 1992; 19; 278-85.

7 Willmott N, Cummings J, Marley E, Smyth JF. Relationship between reductive drug metabolism in tumour tissue of anthracyclines in microspherical form and anti-tumour anthracyclines in microspherical form and
activity. Biochem Pharmacol 1990; 39: 1055-?

8 Harris JR, Canellos GP, Hellman S, Fisher B. In: De Vita

8 Harris JR, Canellos GP, Hellman S, Fisher B. In: De Vita phia: Lippincott, 1985; p 1156.
9 Twelves CJ, Chaudray MA, Reidy J, et al. Toxicity of intra-arterial doxorubicin in locally advanced breast cancer. Cancer Chemother Pharmacol 1990; 25: 459-62.

10 McCarter DHA, Doughty JC, McArdle CS, Cooke TC Reid AW. Angiographic embolisation of the distal internal mammary artery as an adjunct to regional chemotherapy in cular Intervent Radiol, in press.

\title{
Anomalous splenic artery aneurysm: demonstration on CT scanning and angiography
}

\author{
Paul S Sidhu, Kok-Tee Khaw, Anna-Maria Belli
}

\begin{abstract}
Summary
An asymptomatic 35-year-old female was found to have a congenital aneursym of an anomalous splenic artery arising from the superior mesenteric artery on routine investigation for infertility. Demonstration on CT and angiography is presented and the pathogenesis, natural history, management, and implications during pregnancy are discussed.
\end{abstract}

Keywords: splenic artery, aneurism, CT scanning, angiography

\section{Introduction}

Aneurysms arising from the splenic artery are uncommon and are usually removed surgically or embolised to prevent the occurrence of rupture and sudden death. The splenic artery has its usual origin from the coeliac axis but rarely arises from other sites including the superior mesenteric artery (SMA). We describe a case of an anomalous splenic artery derived from the SMA with a congenital saccular aneurysm arising from it.

\section{Case report}

Department of Diagnostic Radiology, Hammersmith Hospital, DuCane Road, London W12 OHS, UK

PS Sidhu A-M Belli

\section{Radiology} Department, Ealing Hospital, London, UK K-T Khaw

\section{Correspondence to} Dr PS Sidhu, 1 A Coleraine Road, Blackheath, London SE3 7PF, UK
An asymptomatic 35-year-old Asian female patient with mild idiopathic thrombocytopaenia purpura (ITP) attended the haematology clinic on a six-monthly basis. The patient was anaemic $(10.3 \mathrm{~g} / \mathrm{dl})$ with a mildly low platelet count $\left(114 \times 10^{9} / 1\right)$ and no other overt manifestations or symptoms attributable to the diagnosis of ITP. Clinical examination of the patient was entirely normal. The patient did, however, complain of infertility. As part of the routine investigations for infertility the patient had an abdominal/pelvic ultrasound. On the B-mode ultrasound examination, a 2-cm rounded echo-poor area thought to be contiguous with the SMA was demonstrated. A subsequent CT scan (Toshiba Xpeed) demonstrated a $2-\mathrm{cm}$ rounded area arising from the
$A$

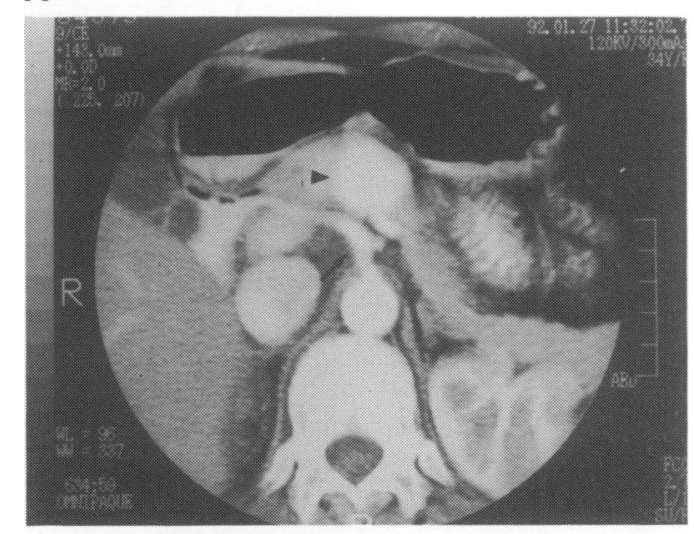

B

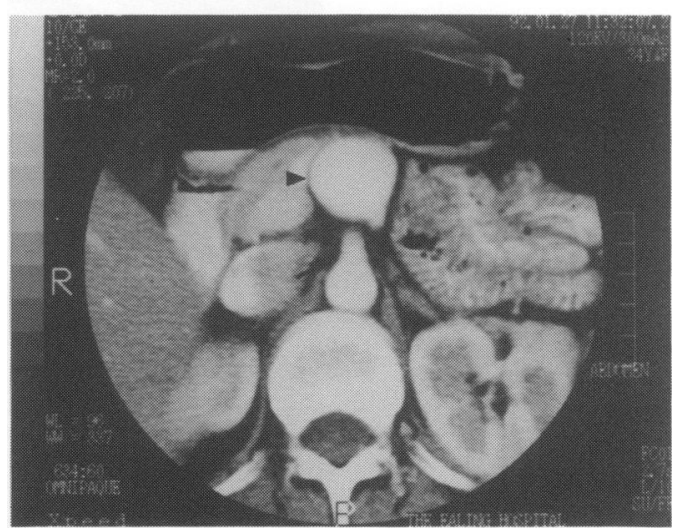

Figure 1 (A) Magnified view of a transaxial section at the level of the coeliac axis (arrow) demonstrating the common hepatic artery (arrow) and a $2-\mathrm{cm}$ vascular aneurysm lying anterior (arrowhead). No splenic artery arises from the coeliac axis. (B) $10 \mathrm{~mm}$ caudal to the level in (A); the origin of the SMA (arrow) and the vascular aneurysm (arrowhead) are seen

splenic artery with uniform enhancement after the administration of contrast (Omnipaque 300) compatible with a vascular aneurysm (figure 1A and B). A Digital Subtraction Angiogram (DSA) performed via selective catheterisation of the SMA, demonstrated an 
anomalous splenic artery arising from the SMA. Adjacent to the anomalous origin of the splenic artery a $2-\mathrm{cm}$ aneurysm was seen (figure $2 A$ and B). Catheterisation of the coeliac axis showed the common hepatic artery and left

A

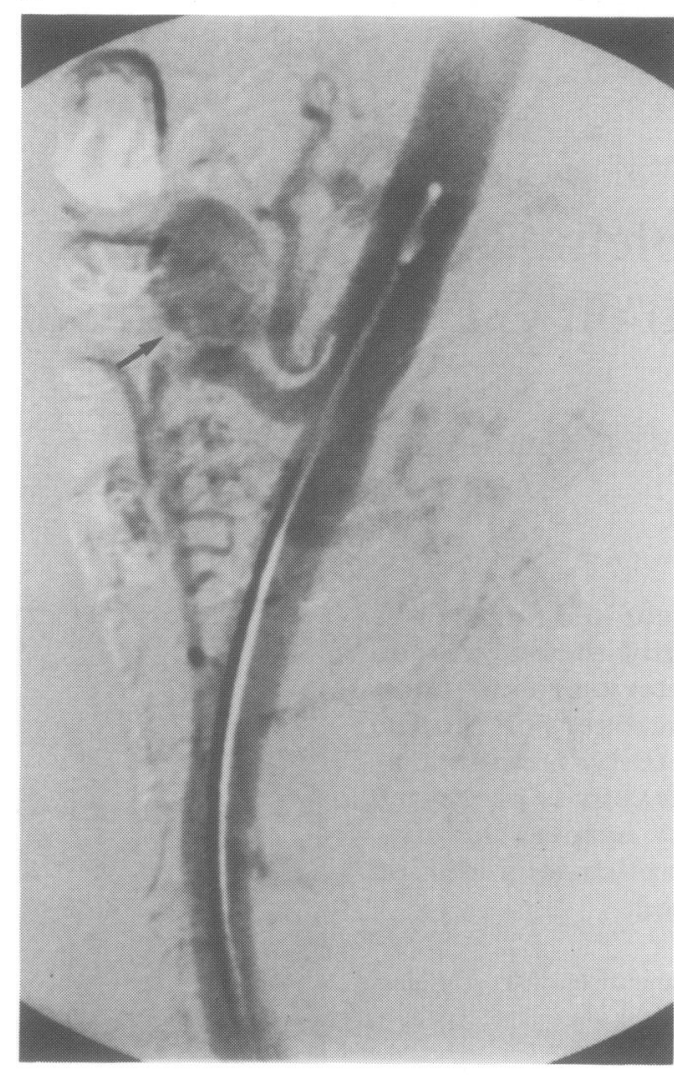

B

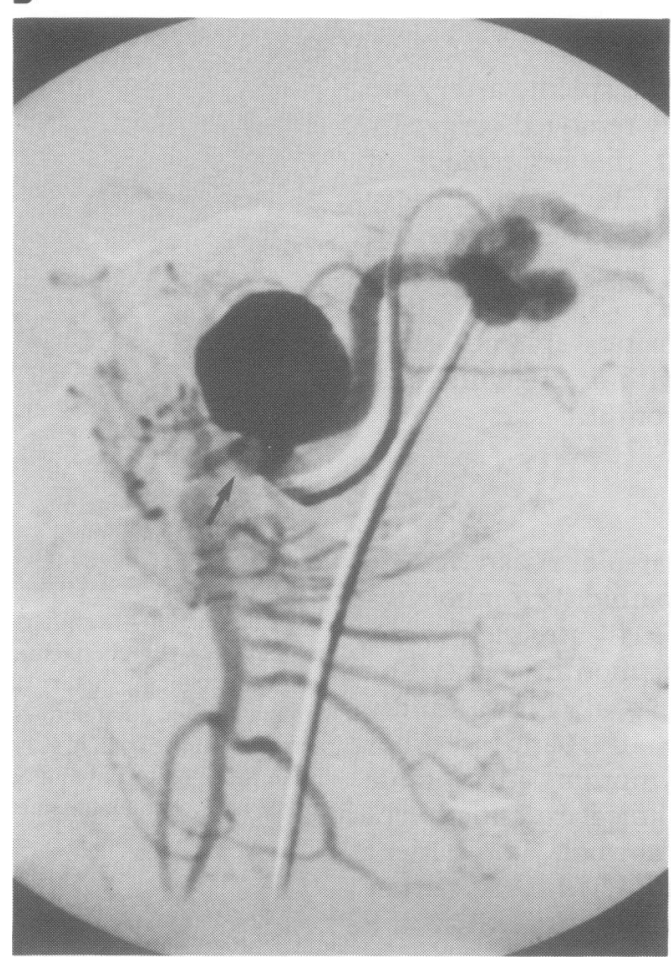

Figure 2 (A) Lateral flush aortogram demonstrates the aneurysm filling from the SMA (arrow). (B) Selective catheterisation of the SMA demonstrates the aneurysm arising at the level of the junction of the origin of the anomalous splenic artery (arrow) as it arises from the SMA gastric artery to arise normally with no evidence of an artery supplying the spleen arising from the coeliac axis. The spleen was of normal size. At laparotomy these findings were confirmed and a $2-\mathrm{cm}$ thin-walled aneurysm 0 arising at the origin of the anomalous splenic of artery was successfully resected. Histological $\frac{\mathrm{C}}{7}$ examination showed a saccular aneurysm with thinning of the media and intima without $z$ significant atheromatous deposits or $\stackrel{\AA}{\varrho}$

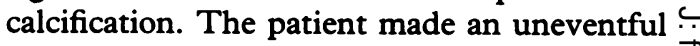
recovery and remains well.

\section{Discussion}

The splenic artery may arise directly from the $\overparen{\otimes}$ aorta, the superior mesenteric, the middle colic, the left gastric, the left hepatic, the right ${ }^{\text {s }}$ hepatic or the common hepatic arteries. ${ }^{1}$ When the coeliac axis has two branches (the left $\overrightarrow{\vec{\omega}}$ gastric and the hepatic arteries) the splenic o artery, as a rule, is derived from the SMA forming a lienomesenteric trunk. In a series of 100 dissections $^{1}$ the splenic artery was described as arising directly from the SMA 'occasionally' at less than $1 \%$. 'Rarely' $(0.8 \%) \stackrel{\omega}{.}$ the splenic artery was duplicated.

Splenic artery aneurysms are generally considered to be uncommon (see box)., ${ }^{2,3}$

These lesions are usually saccular and occur most often at bifurcations of the distal splenic vessel, being multiple in $20 \%$ of patients. The propensity for aneurysmal development had $\vec{\imath}$ been variously attributed to acquired $c$ derangements of the vessel wall, ${ }^{3}$ portal hypertension with splenomegaly, ${ }^{4}$ vascular effects of repeated pregnancy, probably resulting from increased splenic artery shunting during pregnancy. ${ }^{2,3,5}$ This may account for the unusual female:male ratio of $4: 1 .^{6}$

Splenic artery aneurysms are usually asymptomatic, but in two large series up to $20 \%$ of patients had symptoms referable to these lesions. ${ }^{2,3}$ Symptoms include vague left upper quadrant or epigastric pain with occasional radiation to the left scapular region. Most aneurysms are less than $2 \mathrm{~cm}$ and consequently impalpable. The usual mode of presentation of $\dot{\delta}$ splenic artery aneurysms is due to rupture with intraperitoneal haemorrhage and may even 우 mimic common obstetric emergencies in pregnant patients. ${ }^{8}$

\begin{tabular}{|l|l|}
\hline Splenic artery aneurysm & \\
\hline - $0.78 \%$ population \\
- $22 \%$ present as emergencies \\
- $4.5 \%$ remale: 1 male
\end{tabular}


Surgical therapy of splenic artery aneurysms is the treatment of choice for these patients but transcatheter embolisation of the aneurysms may be performed in patients unsuitable for surgery. ${ }^{9}$ Symptomatic aneurysms warrant early surgical therapy as do those encountered in pregnant patients as mortality with aneurysmal rupture during pregnancy is approximately $70 \%$ and fetal mortality approaches $95 \%{ }^{3,8}$ A point of particular importance with this patient whose initial ultrasound examination, when the aneurysm was detected, was in the course of routine investigations for infertility. Operative mortality following surgical treatment for aneurysmal rupture is less than $25 \% \cdot{ }^{10}$ Rupture has been reported to affect between 3 and $9.6 \%$ of all splenic artery aneurysms ${ }^{2,3}$ and as mortality after rupture is high, elective surgery is usually undertaken when an aneurysm is discovered on routine investigation for other symptoms.

An unusual aspect of the splenic aneurysm we

1 Michels NA. Blood supply and anatomy of the upper abdominal organs with a descriptive text. Philadelphia: JB Lippincott Co, 1955; pp 205-8.

2 Stanley JC. Abdominal visceral aneurysms. In: Haimovici H, ed. Vascular emergencies. New York: Appleton-CenturyCrofts 1981; pp 387-96.

3 Stanley JC, Fry WJ. Pathogenesis and clinical significance of splenic artery aneurysms. Surgery 1974; 76: 898-909.

4 Feist JH, Gajaraj A. Extra and intrasplenic artery aneurysm Feist JH, Gajaraj A. Extra and intrasplenic artery aneurys

5 in portal hypertension. Radiology 1977; 125: $331-34$. Deterling RA. Aneurysm of the
diovasc Surg 1971; 12: 309-22.

6 Stanley JC, Zelenock GB. Splanchnic artery aneurysms. In: Rutterford RB, ed. Vascular surgery, 3rd edn. Philadelphia: WB Saunders Co, 1989; pp 969-82. have described is its site of origin at the proximal bifurcation of the SMA and the anomalous splenic artery. Usually an aneurysm arises at a more distal branching point of the splenic artery. There was no clinical history of previous pancreatitis and the pancreas on the CT scan was normal. The presence of collagen vascular disease, vasculitis and infection were excluded on clinical grounds. Histology demonstrated a saccular aneurysm with thinning of the media and the intima without evidence of atheromatous deposits or calcification. A derangement of the vessel wall possibly as a result of the anomalous origin of the splenic artery, resulting in an aneurysm at this site is postulated, but we are not aware of any other similar case reports.

To the best of our knowledge, this is the first reported case of an asymptomatic congenital aneurysm of an anomalous splenic artery arising from the superior mesenteric artery demonstrated on CT scanning and angiography.

7 Hofer BO, Ryan JA, Freeny PC. Surgical significance of vascular changes in chronic pancreatitis. Surg Gynecol Obstet 1987; 164: 499-505.

8 O'Grady JP, Day EJ, Toole AL, Paust JC. Splenic artery aneurysm rupture in pregnancy. A review and case report. Obstet Gynecol 1977; 50: 627-30.

9 Mandel SR, Jaques PF, Sanofsky S, Mauro MA Nonoperative management of peripancreatic arterial Nonoperative management of peripancreatic arterial
aneurysms. A 10-year experience. Ann Surg 1987; 205: aneury $126-8$.

10 Stanley JC, Thompson NW, Fry WJ. Splanchnic artery aneurysms. Arch Surg 1970; 101: 689-97. 